A RECURSION FORMULA FOR THE POLYNOMIAL SOLUTIONS OF A PARTIAL DIFFERENTIAL EQUATION*

BY C. C. MAcDUFFEE

Let us denote by $O$ the differential operator

$$
\frac{\partial^{n}}{\partial x^{n}}-a_{1} \frac{\partial^{n}}{\partial x^{n-1} \partial y}-a_{2} \frac{\partial^{n}}{\partial x^{n-2} \partial y^{2}}-\cdots-a_{n} \frac{\partial^{n}}{\partial y^{n}},
$$

where the $a$ 's are constants, real or complex. Define

$$
\phi(\lambda)=\lambda^{n}-a_{1} \lambda^{n-1}-a_{2} \lambda^{n-2}-\cdots-a_{n} .
$$

Let $A$ be any $n$th order matrix having $\phi(\lambda)=0$ as its minimum equation, and define $Z=A x+I y$. For any function $F(Z)$,

$$
\frac{\partial^{n} F}{\partial x^{n-t} \partial y^{t}}=\frac{d^{n} F}{d Z^{n}} A^{n-t} \text {. }
$$

Then

$$
O F=\frac{d^{n} F}{d Z^{n}} \phi(A),
$$

and since $\phi(A)=0, F(Z)$ is a solution of $O F=0$. If we can write

$$
F(Z)=f_{1}(x, y) I+f_{2}(x, y) A+\cdots+f_{n}(x, y) A^{n-1},
$$

we have

$$
O F(Z)=\sum_{i=1}^{n} O f_{i}(x, y) A^{i-1}=0 .
$$

Since $A$ satisfies no equation of degree $n-1$,

$$
O f_{i}(x, y)=0 \quad(i=1,2, \cdots, n) .
$$

These results are due to Spampinato. $\dagger$

By a proper choice of the matrix $A$, this theorem of Spampinato leads to an interesting recursion formula for the poly-

* Presented to the Society, November 30, 1935.

$\dagger$ Atti Accademia Lincei, Rendiconti, (6), vol. 21 (1935), pp. 73-76. 
nomial solutions of $O f(x, y)=0$ by means of which all such solutions can be computed with rapidity.

Let

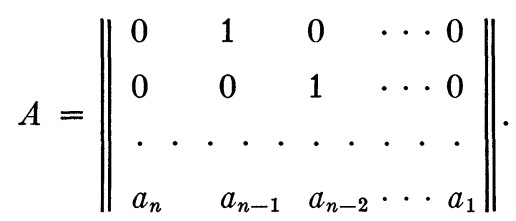

Then $\phi(\lambda)=0$ is the minimum equation of $A$. By means of the relation $\phi(A)=0,(A x+I y)^{k}$ can be written as a linear combination of $I, A, A^{2}, \cdots, A^{n-1}$. The first rows of these matrices are, respectively,

$(1,0,0, \cdots, 0),(0,1,0, \cdots, 0),(0,0,1, \cdots, 0), \cdots,(0,0,0, \cdots, 1)$.

Hence if we denote the first row of $(A x+I y)^{k}$ by $p_{1}{ }^{(k)}, p_{2}{ }^{(k)}, \cdots$, $p_{n}{ }^{(k)}$, we have

$$
(A x+I y)^{k}=p_{1}^{(k)} I+p_{2}^{(k)} A+\cdots+p_{n}^{(k)} A^{n-1} .
$$

If we define the vector

$$
v_{k}=\left(p_{1}^{(k)}, p_{2}^{(k)}, \cdots, p_{n}^{(k)}\right),
$$

it follows from the relation

$$
(A x+I y)^{k+1}=(A x+I y)^{k}(A x+I y),
$$

that

$$
\boldsymbol{v}_{k+1}=\boldsymbol{v}_{k}(A x+I y)
$$

or in full,

$$
\begin{aligned}
& p_{1}^{(k+1)}=y p_{1}^{(k)} \\
& p_{2}^{(k+1)}=x p_{1}^{(k)}+y p_{2}^{(k)} \\
& p_{3}^{(k+1)}=x p_{2}^{(k)}+y p_{3}^{(k)} \\
& p_{n-1}^{(k+1)}= \\
& p_{n}^{(k+1)}= \\
& x p_{n-2}^{(k)}+y p_{n-1}^{(k)}+x a_{2} p_{n}^{(k)}, \\
& x p_{n-1}^{(k)}+\left(y+x a_{1}\right) p_{n}^{(k)},
\end{aligned}
$$

where $p_{1}^{(1)}=y, p_{2}^{(1)}=x, p_{i}^{(1)}=0, i>2$. 
It is evident that $p_{i}{ }^{(k)}$ is a homogeneous polynomial of degree $k$, and by Spampinato's theorem, it is a solution of $O f=0$.

For $k \geqq n$ there are just $n$ linearly independent homogeneous polynomials of degree $k$ which satisfy $O f=0$. For if we let

$$
P_{k}=A_{0} x^{k}+A_{1} x^{k-1} y+\cdots+A_{k} y^{k}
$$

where the $A$ 's are to be determined, then $\partial^{n} P_{k} / \partial x^{n}$ will have the form

$$
h_{1} A_{0} x^{k-n}+h_{2} A_{1} x^{k-n-1} y+\cdots+h_{k} A_{k-n} y^{k-n},
$$

where each $h$ is a positive integer. By forming $O P_{k}$, we obtain a homogeneous polynomial of degree $k-n$ which must vanish identically. This condition leads to $k-n+1$ equations in the $k+1$ unknown $A$ 's. Since the matrix of the coefficients

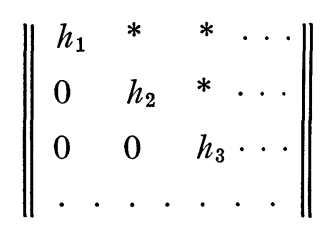

is obviously of rank $k-n+1$, there are exactly $n$ linearly independent solutions.

For $k<n$, the components of $\boldsymbol{v}_{k}$ are, in order, the terms in the binomial expansion of $(y+x)^{k}$ followed by zeros. Hence, for $k<n$, every homogeneous polynomial of degree $k$ is a linear combination of $p_{1}{ }^{(k)}, p_{2}{ }^{(k)}, \cdots, p_{n}{ }^{(k)}$.

We shall prove by induction that for $k \geqq n-1$,

$$
p_{i}{ }^{(k)}=c_{i k} y^{k-i+1} x^{i-1}+\cdots, \quad(i=1,2, \cdots, n),
$$

where the $c_{i k}$ are positive integers and the terms omitted are of degree less than $k-i+1$ in $y$. For $k=n-1$, (2) follows from (1). If we assume (2) to hold for some $k \geqq n-1$, we have from (1)

$$
\begin{aligned}
p_{i}^{(k+1)}= & x p_{i-1}^{(k)}+y p_{i}^{(k)}+x a_{n-i+1} p_{n}^{(k)} \quad\left(p_{0}^{(k)}=0\right) \\
= & x c_{i-1, k} y^{k-i+2} x^{i-2}+y c_{i k} y^{k-i+1} x^{i-1} \\
& +x a_{n-i+1} c_{n k} y^{k-n+1} x^{n-1}+\cdots \\
= & \left(c_{i-1, k}+c_{i k}\right) y^{k-i+2} x^{i-1}+\cdots
\end{aligned}
$$


where the terms represented by . . are of degree less than $k-i+2$ in $y$. Thus (2) is established for $k+1$ and hence by induction for $k \geqq n-1$. Hence $p_{1}{ }^{(k)}, p_{2}{ }^{(k)}, \cdots, p_{n}{ }^{(k)}$ are linearly independent for $k \geqq n-1$.

We have now proved that, for every $k$, every linear homogeneous polynomial of degree $k$ which is a solution of $O f=0$ has the form

$$
c_{1} p_{1}{ }^{(k)}+c_{2} p_{2}{ }^{(k)}+\cdots+c_{n} p_{n}{ }^{(k)},
$$

where the $c$ 's are arbitrary constants.

The University of Wisconsin

\section{A CHARACTERIZATION OF NULL SYSTEMS IN PROJECTIVE SPACE}

\section{BY RICHARD BRAUER}

1. Introduction. We consider the $n$-dimensional projective space $S_{n}$ defined analytically by means of any abstract field $F$. The points $P$ of $S_{n}$ are given by a set of $n+1$ elements $x_{i}$ of $F, P=\left(x_{0}, x_{1}, \cdots, x_{n}\right)$, (not all $\left.x_{i}=0\right)$, with the convention that proportional sets define the same point. The points $P$ whose coordinates satisfy a linear homogeneous equation $u^{(0)} x_{0}+u^{(1)} x_{1}+\cdots+u^{(n)} x_{n}=0$, (not all $u^{(i)}=0$ ), form a hyperplane $\epsilon=\left(u^{(0)}, u^{(1)}, \cdots, u^{(n)}\right)$. There is no difficulty in defining such notions as those of straight lines, projections, and cross ratios, and discussing the elementary properties.

Let $M$ be a non-singular skew-symmetric bilinear form with coefficients $a_{i k}$ in $F$,

$$
M=\sum_{i, k=0}^{n} a_{i k} y_{i} x_{k}, \quad a_{i k}=-a_{k i}, \quad \operatorname{det}\left(a_{i k}\right) \neq 0 .
$$

For every point $P=\left(x_{0}, x_{1}, \cdots, x_{n}\right)$ the equation $M=0$ is the equation of a hyperplane $\epsilon$ in the coordinates $\left(y_{0}, y_{1}, \cdots, y_{n}\right)$ of a variable point of $\epsilon$. We obtain in this manner a one-to-one correspondence between the points $P=\left(x_{0}, x_{1}, \cdots, x_{n}\right)$ and hyperplanes $\epsilon=\left(u^{(0)}, u^{(1)}, \cdots, u^{(n)}\right)$ of $S_{n}$ which is called a null system. The relation between corresponding values of the $u^{(i)}$ and $x_{i}$ is given by 